\title{
Effect of soil moisture on spatial variation of soil heat capacity
}

\author{
Huanzhuang Tao1,a, Siwen Zheng², Ying Lin², Lei Gan ${ }^{*}$ *3,b, \\ Yangjian Peng ${ }^{3}$, Rui Ma ${ }^{3}$, Fangli Cheng ${ }^{4}$
}
${ }^{1}$ Guangxi Collaborative Innovation Center for Water Pollution Control and Water Safety in Karst Area, Guilin University of Technology, Guilin 541004, China;

${ }^{2}$ Guangxi Key Laboratory of Environmental Pollution Control Theory and Technology, Guilin University of Technology, Guilin 541004, China;

${ }^{3}$ College of Environmental Science and Engineering, Guilin University of Technology, Guilin 541004, China;

${ }^{4}$ Guilin Academy of Agricultural Sciences, Guilin 541004, China

a799634972@qq.com, ballen_gl2006@163.com

${ }^{*}$ Corresponding author

Keywords: Soil heat capacity, Soil moisture content, Geostatistical analyst, Spatial distribution

\begin{abstract}
In this research, the variations and spatial distribution of soil heat capacity in soybean field and sugarcane field under drought, moderate and humid soil moisture conditions were studied. The soil heat capacity in order to analyze spatial distribution by geostatistical analyst in each plot covered was calculated by the measured soil moisture content, furthermore, the states of dry, moderate and wet soil moisture conditions were determined. The result showed the soil heat capacity was greatest in sugarcane plot but lowest in soybean plot, which was similar to the change of soil moisture content under three soil moisture conditions. It suggested that the soil water content was a primary factor resulting in the change of the soil heat capacity. According to the results of geostatistical analysis of soil heat capacity, the spatial structure ratio of soil heat capacity is $57 \%$ $-93 \%$ under three soil moisture conditions, which belongs to strong moderate spatial relativity. The high threshold spatial distribution of soil heat capacity in soybean and sugarcane fields is stable under drought and humid conditions, which indicates that the soil itself has the ability to restore the stability of its spatial distribution.
\end{abstract}

\section{Introduction}

Soil heat capacity is one of the soil thermal properties, a basic physical parameters, generally reflecting the soil temperature changed rate, heat retention and transmission capacities [1]. At the same time, the change of temperature field can also cause the potential energy imbalance, to a certain extent, affected the vertical distribution of soil moisture content [2]. Soil heat capacity mainly depends on soil moisture content, and soil heat capacity of different land use types with the increase of soil moisture content increased [3]. Yue et al. [4] studied the soil temperature, humidity and thermal characteristics before and after precipitation in the semi-arid grassland of the Loess Plateau, and proved that the precipitation changed the soil moisture and also made the soil heat capacity changed. Therefore, the study of the spatial variation of soil heat capacity is the basis for the efficient utilization of soil water and nutrient, and also an important aspect to improve soil quality. In this research, the main study region is Guilin Academy of Agricultural Sciences, Guilin, China. The variation and the spatial distribution of soil heat capacity under drought, moderate and 
humid soil conditions were studied in soybean and sugarcane land use types. This is of great significance to the reasonable adjustment of the soil condition.

\section{Materials and methods}

\subsection{General situation of test area}

The experimental area is located in the Guilin Academy of Agricultural Sciences $\left(25^{\circ} 4^{\prime} \mathrm{N}\right.$; $109^{\circ} 44^{\prime} \mathrm{E}$ ) in Guilin, China, and with the subtropical monsoon climate. The mean annual rainfall in the area is about $1894 \mathrm{~mm}$, with $60 \%$ to $80 \%$ of the precipitation occurring between April and September each year. The mean evaporation is between $1490 \mathrm{~mm}$ and $1905 \mathrm{~mm}$, and the mean annual temperature is $18{ }^{\circ} \mathrm{C}$. The area of $135 \mathrm{~m} \times 105 \mathrm{~m}$ was selected using hand held GPS in soybean planting area and sugarcane planting area respectively, and every $15 \mathrm{~m}$ set one measuring point, totaling 80 points. At the same time, 20 subordinate measuring points were randomly set in each experimental areas, the entire test area set a total of 100 measurement points.

\subsection{Sampling and analysis}

Prior to the start of the test, samples were collected from two test areas $(0-6 \mathrm{~cm})$ and taken to the laboratory for determination of soil texture, bulk density, total porosity, and organic matter. The soil moisture content $(0-6 \mathrm{~cm})$ at each measurement point was measured by a HH2 Moisture Meter (Theta-probe Type ML2x). Measurement time is from August 2014 to March 2015. Based on the analysis of the soil moisture content under different land use types, the soil heat capacity of three different soil moisture states of drought, medium and humid were analyzed and discussed.

According to De Vries method to calculate the soil heat capacity, the calculation formula as follows [5]:

$$
C_{\mathrm{s}}(\theta)=\theta+0.46\left(1-\phi-m_{\mathrm{o}}\right)+0.6 m_{\mathrm{o}}
$$

In the formula, $\operatorname{Cs}(\theta)$ is soil heat capacity $\left(\mathrm{cal} / \mathrm{cm}^{3} \cdot{ }^{\circ} \mathrm{C}\right), \theta$ is soil water content $\left(\mathrm{cm}^{3} / \mathrm{cm}^{3}\right)$, $\varnothing$ is total porosity, $m_{o}$ is organic fractions, 0.46 and 0.6 are volumetric heat capacity of mineral substance and soil organic matter respectively $\left(\mathrm{cal} / \mathrm{cm}^{3} \cdot{ }^{\circ} \mathrm{C}\right)$.

In geostatistical analysis of soil heat capacity, the method that using half variant function analyzed the data space changes, and obtain important parameters such as Nugget $\left(C_{0}\right)$, Partial Sill $(C)$, Sill $\left(C+C_{0}\right)$, and maximum correlation range. The nugget represents the heterogeneity of the random part and determines the size of the variation. The sill value includes structural variance and random variance, which is the limit of the semivariogram function [6]. The spatial correlation can be represented by the spatial structure ratio, that is, the ratio of the partial sill $(C)$ to the sill $(C+$ $C_{0}$ ), and the larger the value is, the stronger the spatial correlation is. According to the study of Cambardella et al. [7], the spatial correlation between variables is very strong when the spatial structure ratio is greater than $75 \%$; and the spatial correlation between the variables is moderate when the spatial structure ratio is $25 \%$ to $75 \%$; the spatial correlation of the variables is very weak when the spatial structure ratio is less than $25 \%$. All the data were calculated and analyzed by SPSS 13.0 (SPSS Inc., Chicago, USA). Geostatistical analysis and spatial distribution mapping of soil heat capacity were performed with Arcgis 10.0 (ESRI, USA) software.

\section{Result and analysis}

\subsection{The soil properties}

Table 1 The soil texture, bulk density (BD), total porosity and soil organic matter (SOM), in 2 plots

\begin{tabular}{|c|c|c|c|c|c|c|}
\hline \multirow[t]{2}{*}{ plot } & \multicolumn{3}{|c|}{ texture } & $\mathrm{BD} /$ & $\mathrm{TP} /$ & SOM/ \\
\hline & Sand/ (\%) & Silt/（\%) & Clay/ (\%) & $\left(\mathrm{g} \cdot \mathrm{cm}^{-3}\right)$ & (\%) & $\left(\mathrm{g} \cdot \mathrm{kg}^{-1}\right)$ \\
\hline Soybean & $37.11 \pm 1.7 a$ & $25.68 \pm 1.3 a$ & $37.21 \pm 2.1 \mathrm{a}$ & $1.38 \pm 0.02 a$ & $45.17 \pm 0.7 a$ & $11.41 \pm 0.25 a$ \\
\hline Sugarcane & $35.67 \pm 2.1 \mathrm{a}$ & $26.28 \pm 1.8 \mathrm{a}$ & $38.05 \pm 2.2 \mathrm{a}$ & $1.29 \pm 0.04 b$ & $47.78 \pm 0.6 a$ & $13.36 \pm 0.34 \mathrm{a}$ \\
\hline
\end{tabular}

Note: Different lowcase letters indicate the significant difference $(P<0.05), \mathrm{n}=7$.

Table 1 lists the basic properties of the soil in the soybean and sugar cane test areas. There 
were no significant differences in soil texture between the two experimental zones. The soil bulk density was significantly higher in soybean than in sugarcane field $(\mathrm{P}<0.05)$. The total porosity of soybean field and sugarcane field were $45.17 \%$ and $47.78 \%$, with no significant difference. Organic matter content of soybean is lower than that of sugarcane land.

\subsection{Statistical description of Soil moisture content and soil heat capacity}

Table 2 lists the basic statistical descriptions of soil heat capacity and soil moisture in two experimental zones under drought, moderate, and humid conditions. The soil moisture contents in sugarcane land were all higher than those in soybean land under three soil moisture conditions. The soil moisture content of soybean field was the smallest under the drought condition, which was $0.044 \mathrm{~cm}^{3} \cdot \mathrm{cm}^{-3}$. The soil moisture content of sugarcane was the highest under the humid condition, which was $0.226 \mathrm{~cm}^{3} \cdot \mathrm{cm}^{-3}$. Under three soil moisture conditions, the soil heat capacity has the same trend, the value in sugarcane land were all higher than in soybean land, and the minimum is in soybean field under drought condition, which is $0.298 \mathrm{cal} \cdot \mathrm{cm}^{-3} \cdot{ }^{\circ} \mathrm{C}^{-1}$, and the maximum is under humid condition and $0.468 \mathrm{cal} \cdot \mathrm{cm}^{-3} \cdot{ }^{\circ} \mathrm{C}-1$. The moisture content of sugarcane is higher than that of soybean. The reason is that adopting the furrow irrigation method in the sugarcane field could store much more moisture, and its bare soil area is less than that of the soybean field, so the soil moisture content of the sugarcane field is slightly higher than the soybean land, and then the soil heat capacity is larger than that of soybean land.

Table 2 Soil moisture content and soil volumetric heat capacity under three moisture conditions

\begin{tabular}{|c|c|c|c|c|c|}
\hline $\begin{array}{c}\text { Moisture } \\
\text { state }\end{array}$ & Plot & $\begin{array}{c}\text { Moisture content mean } \\
\left(\mathrm{cm}^{3} \cdot \mathrm{cm}^{-3}\right)\end{array}$ & $\begin{array}{c}\text { Standard error } \\
\left(\mathrm{cm}^{3} \cdot \mathrm{cm}^{-3}\right)\end{array}$ & $\begin{array}{c}\text { Heat capacity mean } \\
\left(\mathrm{cal} \cdot \mathrm{cm}^{-3} \cdot{ }^{\circ} \mathrm{C}^{-1}\right)\end{array}$ & $\begin{array}{c}\text { Standard error } \\
\left(\mathrm{cal} \cdot \mathrm{cm}^{-3} \cdot{ }^{\circ} \mathrm{C}^{-1}\right)\end{array}$ \\
\hline \multirow{2}{*}{ Drought } & Soybean & 0.044 & 0.002 & 0.298 & 0.002 \\
\cline { 2 - 6 } & Sugarcane & 0.058 & 0.002 & 0.300 & 0.002 \\
\hline \multirow{2}{*}{ Moderate } & Soybean & 0.121 & 0.004 & 0.375 & 0.004 \\
\cline { 2 - 6 } & Sugarcane & 0.149 & 0.004 & 0.391 & 0.004 \\
\hline \multirow{2}{*}{ Humid } & Soybean & 0.163 & 0.003 & 0.417 & 0.003 \\
\cline { 2 - 6 } & Sugarcane & 0.226 & 0.006 & 0.468 & 0.006 \\
\hline
\end{tabular}

\subsection{Soil heat capacity of the statistical analysis}

Table 3 The geostatistics results of soil volumetric heat capacity under three moisture conditions

\begin{tabular}{|c|c|c|c|c|c|c|}
\hline $\begin{array}{c}\text { Moisture } \\
\text { state }\end{array}$ & Plot & $\begin{array}{c}\text { Range } \\
(\mathrm{m})\end{array}$ & Nugget & $\begin{array}{c}\text { Partial } \\
\text { Sill }\end{array}$ & Sill & $\begin{array}{c}\text { Spatial structure ratio } \\
(\%)\end{array}$ \\
\hline \multirow{2}{*}{ Drought } & Soybean & 27.5 & $8.11 \mathrm{E}-05$ & $1.41 \mathrm{E}-04$ & $2.22 \mathrm{E}-04$ & 63.46 \\
& Sugarcane & 70.05 & $8.62 \mathrm{E}-05$ & $1.33 \mathrm{E}-04$ & $2.19 \mathrm{E}-04$ & 60.69 \\
Moderate & Soybean & 34.64 & $9.29 \mathrm{E}-05$ & $1.18 \mathrm{E}-03$ & $1.28 \mathrm{E}-03$ & 92.72 \\
& Sugarcane & 207.2 & $3.19 \mathrm{E}-04$ & $1.52 \mathrm{E}-03$ & $1.84 \mathrm{E}-03$ & 82.61 \\
Humid & Soybean & 103.73 & $4.70 \mathrm{E}-04$ & $6.25 \mathrm{E}-04$ & $1.10 \mathrm{E}-03$ & 57.12 \\
& Sugarcane & 207.2 & $2.94 \mathrm{E}-03$ & $5.63 \mathrm{E}-04$ & $3.51 \mathrm{E}-03$ & 16.06 \\
\hline
\end{tabular}

Table 3 shows the results of geostatistical analysis of soil heat capacity under three soil moisture conditions in soybean and sugarcane experimental areas. Under the three conditions, the maximum correlation range of the soil heat capacity are in the range of 27.50-207.20 m, which is larger than the distance between the observed points of the test area, proving the spatial correlation between these observations. The nugget value of the two experimental areas are basically less than partial sill, only in Sugarcane under humid condition, the nugget is greater than the partial sill. At the same time, soybean under drought condition, nugget and partial sill difference is big, and under the condition of moderate and humid nugget and partial sill difference is very small, which indicated that the spatial variability of soil heat capacity in soybean field might be affected by the combined effects of atmospheric rainfall and man-made farming factors. From spatial structure ratio, the spatial structure ratio of soybean and sugarcane was more than $25 \%$, which was moderate spatial correlation under drought and humid condition. But under moderate moisture conditions, the 
spatial structure ratio of soybean and sugarcane is more than $75 \%$, indicating that it has strong spatial correlation.

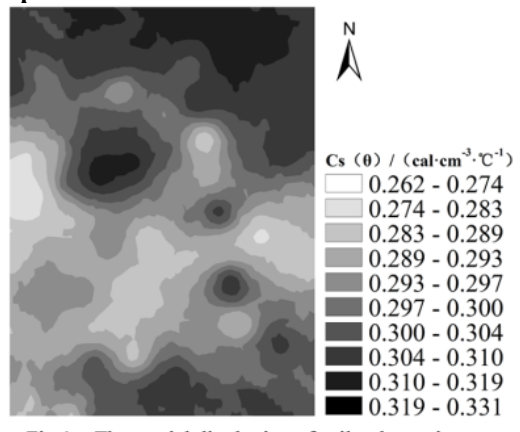

Fig.1-a The spatial distrbution of soil volumetric heat capacity under drought condition in Soybean field

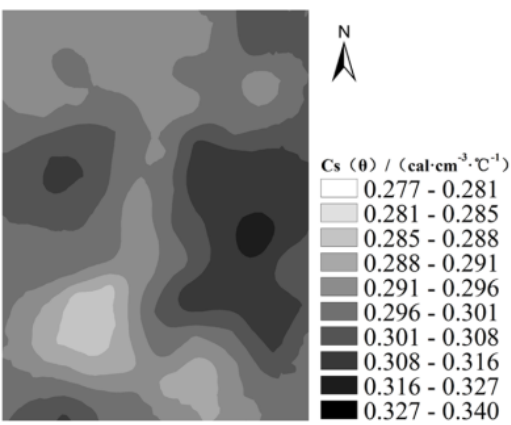

Fig.2-a The spatial distrbution of soil volumetric

heat capacity under drought condition in Sugarcane field

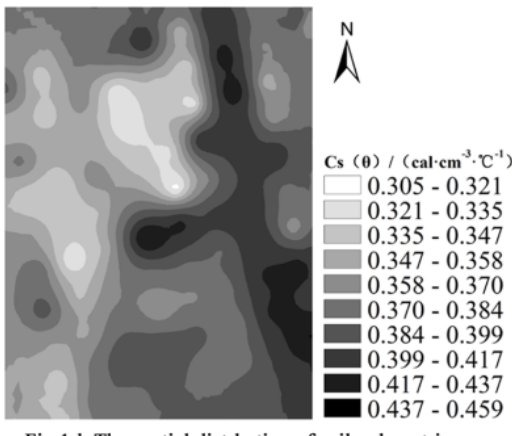

Fig.1-b The spatial distrbution of soil volumetric heat capacity under medium condition in Soybean field

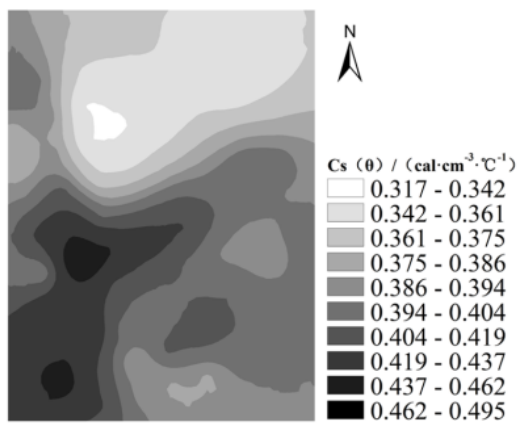

Fig.2-b The spatial distrbution of soil volumetric

heat capacity under medium condition in Sugarcane field

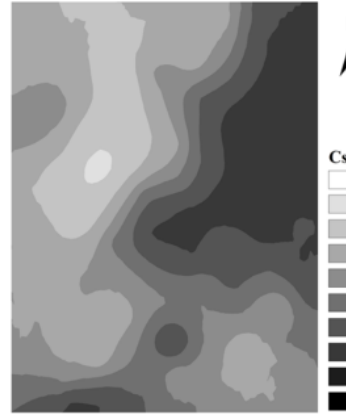

$\bigwedge^{N}$

Fig.1-c The spatial distrbution of soil volumetric

heat capacity under humid condition in Soybean field

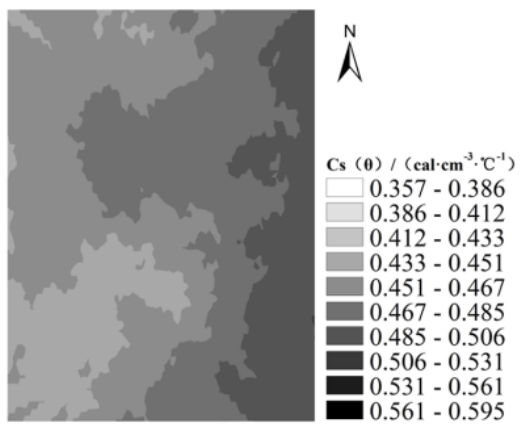

Fig.2-c The spatial distrbution of soil volumetric

heat capacity under humid condition in Sugarcane field

The spatial distribution of soil heat capacity in two experimental zones under different soil moisture conditions was obtained by using the Kriging interpolation method in Arcgis for geostatistical analysis of soil heat capacity. The shades of each figure represents the value of soil heat capacity, the darker the color, the higher the soil heat capacity, the shade of each picture changes have their own changed threshold. Fig.1-a, Fig.1-b and Fig.1-c represent the spatial distribution of soil heat capacity in soybean field under drought, moderate and humid soil moisture conditions, respectively. In the medium moisture condition, the high threshold is located in the southeast corner, while the drought and humid conditions, it is located in the northeast corner of the test area. Mainly affected by artificial cultivation and irrigation, resulting in uneven distribution of soil moisture content, the corresponding distribution of soil heat capacity would be chaotic. Fig.2-a, Fig.2-b and Fig.2-c represent the spatial distribution of soil heat capacity in Sugarcane field under drought, moderate and humid soil moisture conditions, respectively. In the moderate condition, the high threshold of the soil heat capacity is located in the southwest corner, but under the drought and humid conditions, the high threshold is distributed in the east of the test area. The main causes of these changes are human factors by planting sugarcane in the furrow irrigation method used in the trench storage of water, combined with the impact of rainfall, making the soil moisture distribution uneven, thus influenced the spatial distribution of heat capacity. In general, the spatial variability of soil heat capacity under different soil moisture conditions in the two experimental zones were different, but their high threshold were stable under drought and humid conditions, which indicated that the soil had its own recovery spatial distribution stability ability.

\section{Conclusion and discussion}

The soil heat capacity was greatest in sugarcane plot but lowest in soybean plot, which was similar to the change of soil moisture content under three soil moisture conditions. It suggested that the soil water content was a primary factor resulting in the change of the soil heat capacity. According to the results of geostatistical analysis of soil heat capacity, the spatial structure ratio of soil heat capacity is $25 \%-75 \%$, which was moderate spatial correlation under drought and humid conditions. But under moderate moisture conditions, the spatial structure ratio of soybean and sugarcane is more than $75 \%$, indicating that it has strong spatial correlation. The spatial variation of 
soil heat capacity is controlled by natural factors such as rainfall and human factors such as irrigation. The soil moisture content is the main factor that causes the spatial variation of soil heat capacity. Although the spatial distribution of soil heat capacity in soybean and sugarcane fields was different under moderate condition, their spatial distribution was stable under drought and humid conditions, indicating that the soil itself had the ability to restore its spatial distribution stability.

\section{Acknowledgements}

The author thanks the support of the Natural Science Foundation of China (No.41501230), Natural Science Foundation of Guangxi (No.2016GXNSFAA380197), and Natural Science Foundation of Guangxi (No.2014GXNSFBA118098).

\section{References}

[1]Tyson, E.O., Horton, et al. A new perspective on soil thermal properties. Soil Sci. Soc. Am. J. (2001) 65, 1641-1647.

[2]Li Huixing , Xia Ziqiang, Ma Guanghui. Effects of water content variation on soil temperature process and water exchange. Journal of Hohai University. (2007)02:172-175.

[3]Gan L, Peng X, Peth S,et al. Effects of grazing intensity on soil thermal properties and heat flux under Leymus chinensis and Stipa grandis vegetation in Inner Mongolia, China. Soil and Tillage Research. (2012)118(5):147-158.

[4]Yue Ping, Zhang Qiang, Wang Sheng, et al. Characteristics of soil temperature, moisture and heat pro- and post- precipitation in semiarid grassland over LongZhong Loess Plateau. Journal of Desert Research. (2013)06:1766-1774.

[5]De Vries, D.A., Thermal properties of soils, in Physics of plant environment (2nd ed.). In: W. R. van Wijk, Editor, Physics of Plant Environment, North Holland Publishing Company, Amsterdam. (1966)pp. 210-235.

[6] Wang Lixia, Duan Wenbiao, Chen Lixin, et al.Effects of gap size on the spatial heterogeneity of soil water in Pinus koraiensis-dominated broad-leaved mixed forest. Chinese Journal of Applied Ecology, Jan. (2013)24(1) : 17-24.

[7]Cambardella C A, Moorman T B, Parkin T B, et al. Field scale variability of soil properties in Central Iowa soils. Soil Science Society America Journal. (1994)58: 1501-1511. 